\title{
Cartilage-on-a-chip to aid OA drug development
}

chondrocytes
grown under
hyper-
physiological
compression
for 7 days
showed a
distinct OA-like
phenotype

The development of diseasemodifying osteoarthritis (OA) drugs (DMOADs) has been hampered by a lack of experimental models that accurately recapitulate the disease phenotype. However, a new cartilage-on-a-chip device looks set to open up the possibility of high-throughput drug screening for OA.

"The conceptual design was inspired by a previous beating hearton-chip device," explains last author Marco Rasponi. "The device consists of a top cell culture chamber in which a 3D cell-laden hydrogel is confined by two rows of overhanging posts in a miniaturized rectangular channel. This chamber lies on a membrane covering a pneumatic chamber. As the pressure in the pneumatic chamber increases, the membrane bends upward and compresses the hydrogel while the hanging posts limit the lateral expansion."

The researchers first used this device to culture adult human articular chondrocytes in a synthetic polyethylene glycol hydrogel,

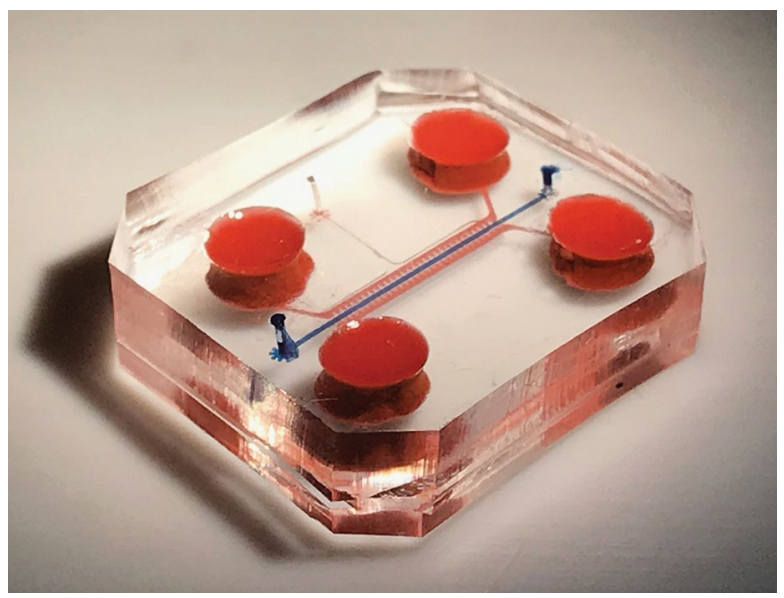

Photograph of an assembled device. Adapted from Occhetta, P. et al. Nat. Biomed. Eng. 3, 545-557 (2019) thereby creating a microtissue, or cartilage-on-a-chip. "A cartilaginous construct was achieved in the device following 14 days of chondrogenic differentiation in a 3D environment, and was characterized by the deposition of extracellular matrix rich in type II collagen and aggrecan, two major constituents of adult cartilage," states co-first author Andrea Mainardi.

Evidence that abnormal joint loading and biomechanics can influence the development of $\mathrm{OA}$ led the researchers to add cyclical rounds of compression to the culture conditions to try and create OA-like cartilage. Two different levels of compression were used: $10 \%$ (physiological compression) and 30\% (hyper-physiological compression). Chondrocytes grown under hyper-physiological compression for 7 days showed a distinct OA-like phenotype at a genetic and cellular level.

"A major finding of this paper is that mechanical hyper-physiological compression alone is somehow sufficient to elicit OA-like traits in a cartilaginous construct," says corresponding author Andrea Barbero. "The OA-like traits were achieved without the need for the administration of cytokines, which are often used in OA in vitro models."

"Despite limitations related to its focus on cartilage, rather than the whole joint, this model is more effective than other ex vivo and in vitro models that rely on single stimuli such as a pro-inflammatory cytokine," says Mary Goldring, an expert in OA who was not involved in this study. "Indeed, using this model it is possible to observe a whole constellation of responses and network interactions involved in promoting the inflammatory response in cartilage, the catabolicanabolic imbalance and the hypertrophic phenotype that usually cannot be assessed together."

Having established an OA-like model, the researchers tested the effects of anakinra, rapamycin and celecoxib (drugs known to counteract OA-associated inflammation) against dexamethasone as a reference compound, all of which behaved as expected from previous in vivo studies. The hyaluronic acid alkylamide $\mathrm{HYADD}^{\circledR} 4$, which is currently being developed for use in $\mathrm{OA}$, was also tested against hyaluronic acid as a reference, and reduced matrix metalloproteinase expression in a manner consistent with the results of preclinical studies.

In the future, the authors hope to further develop the device for high-throughput use. "Including new features such as the introduction of tissues other than cartilage in the model to make it better resemble the whole joint is another option we are looking into," adds Barbero.

"One could imagine that the availability of different compartments might enable the development of a 'joint-on-a-chip', although the introduction of such complexity by, for example, adding a subchondral bone compartment along with synovium and other peri-articular tissues might reduce the ability to show efficacy of a single DMOAD candidate," concludes Goldring. "Nevertheless, one could envision, as a next step, having a two-compartment system with a 'cartilage-synovium co-culture-ona-chip', since interactions between these two tissues are important for promoting disease progression in post-traumatic OA."

Joanna Collison

ORIGINAL ARTICLE Occhetta, P. et al. Hyperphysiological compression of articular cartilage induces an osteoarthritic phenotype in a cartilage-on-a-chip model. Nat. Biomed. Eng. 3, 545-557 (2019) 